\title{
Detección precoz de EPOC en las farmacias comunitarias de Baleares (FARBALEPOC)
}

\author{
Francesc Moranta Ribas' ${ }^{1}$ Lucía Gorreto López² \\ 1. Farmacéutico comunitario en Palma de Mallorca. Presidente de la delegación balear de la Sociedad Española de Farmacia Familiar y Comunitaria \\ (SEFAC). Miembro del grupo de Respiratorio de SEFAC. 2. Médico de Familia en Mallorca. Centro de Salud del Arenal. Servei Balear de Salut. Integrante \\ del grupo de Patologías del sistema respiratorio de IBAMFIC. Coordinadora del grupo de Tabaquismo de IBAMFIC.
}

\section{PALABRAS CLAVE}

EPOC, espirometría, farmacia comunitaria, cribado

\section{ABREVIATURAS}

AP: atención primaria. COPD-PS: Cuestionario validado usado para el cribado de EPOC. EPOC: enfermedad pulmonar obstructiva crónica.

FEV1: volumen espiratorio forzado en un segundo. FEV1/FEV6: relación para establecer posible paciente de EPOC por obstrucción al flujo aéreo.

FEV6: volumen espiratorio forzado en seis segundos. IBAMFIC: Societat Balear de Medicina Familiar i Comunitària.

SEFAC: Sociedad Española de Farmacia Familiar y

Comunitaria.

SEPAR: Sociedad Española de Neumología y Cirugía Torácica.

\section{KEYWORDS}

COPD, spirometry, community pharmacy, screening

\section{RESUMEN}

Introducción: Importantes estudios como el EPISCAN ha puesto de manifiesto en España, el retraso diagnóstico de la EPOC y una elevada tasa de infradiagnóstico, encontrando que el 73\% de los pacientes estaba sin diagnosticar y en la mayoría de los que se diagnosticaban ya se encontraban en estadios III y IV. Este retraso en el diagnóstico provoca una elevada morbilidad, así como un elevado coste asistencial. Por todo esto es necesario un diagnóstico precoz.

Objetivos: Identificar pacientes de EPOC no diagnosticados, mediante un servicio de cribado de EPOC ofrecido por farmacias comunitarias.

Material y métodos: Población de estudio: mayores de 40 años de ambos sexos, fumadores, exfumadores, y fumadores pasivos, que presentan signos o síntomas crónicos, y que en el cuestionario COPD-PS obtengan una puntuación $\geq 4$. A los pacientes se les realizó la prueba de espiración forzada mediante el dispositivo portátil Vitalograph COPD-6. Si presentaba valores del cociente FEV1/FEV $6<0,75$ se derivaba el paciente a su médico de familia para que se le realizase la espirometría convencional de confirmación.

Resultados: Se encuestaron un total de 198 pacientes, 154 de ellos presentaban COPD-PS $\geq 4,38$ $(24,7 \%)$ de ellos presentaron resultados del cociente FEV1/FEV6 $<0,75$. La prevalencia de posible EPOC en este estudio se ha estimado en un 19,2\% de los pacientes encuestados.

Conclusiones: El significativo porcentaje de pacientes a los se les ha detectado una posible EPOC, nos demuestra la utilidad de implantar servicios de cribado y detección precoz de EPOC en las farmacias comunitarias.

\section{Early detection of COPD in community pharmacies in the Balearic Islands (FARBALEPOC)}

\section{ABSTRACT}

Introduction: Important studies, as EPISCAN, have revealed in Spain a delay in diagnosis of COPD and a high rate of underdiagnosis, finding that 73\% of patients were undiagnosed and most of those who were already diagnosed were in III and IV stages of COPD. This delay in diagnosis causes high morbidity and high health care costs. For all that, early diagnosis is necessary.

Objectives: To identify undiagnosed COPD patients, using a COPD screening service offered by community pharmacies.

Material and methods: Study Population: current, former and passive smokers, aged 40 or older who have signs or chronic symptoms were recruited. Those who obtained a score $\geq 4$ in COPD-PS questionnaire underwent a forced expiratory test by using Vitalograph COPD-6 portable device. If the FEV 1 $\mathrm{FEV}_{6}$ value was $<0.75$, the patient was referred to the general practitioner (GP) in order to confirm COPD diagnosis with conventional spirometry.

Results: A total of 198 patients were recruited, 154 of them had a COPD-PS score $\geq 4$, of whom 38 $(24.67 \%)$ presented results of $\mathrm{FEV}_{1} / \mathrm{FEV}_{6}<0.75$. The prevalence of COPD in this study may have been estimated at $19.2 \%$ of patients enrolled.

Discussion and conclusions: The significant proportion of patients detected with a possible diagnosis of COPD demonstrates the utility to implement services of screening and early detection of COPD in community pharmacies.

Parte de este trabajo se presentó como póster en el VI Congreso Nacional de Farmacéuticos Comunitarios, Málaga 2014. 


\section{Introducción}

La enfermedad pulmonar obstructiva crónica (EPOC) es una enfermedad crónica y progresiva que se caracteriza por la obstrucción poco reversible al flujo aéreo, como consecuencia de la exposición activa o pasiva a gases nocivos (en el mundo occidental, principalmente el humo del tabaco).

Actualmente es la cuarta causa de muerte a nivel mundial. En nuestro país, aproximadamente el $1 \%$ de la población general padece EPOC, aumentando hasta el 10-20\% en muestras de mayores de 40 años. La prevalencia para el grupo de 40-80 años es del 10,2\%, un 90\% de ellos son fumadores o exfumadores. Se acepta que el tabaco es el principal factor de riesgo y que puede afectar a un 20-25\% de los fumadores $(1,2)$.

$\mathrm{Su}$ detección en estadios precoces, unido al abandono del hábito tabáquico, pueden comportar un mejor pronóstico. Importantes estudios, primero el estudio IBERPOC (1) y posteriormente el estudio EPISCAN (2), han puesto de manifiesto el retraso diagnóstico de la EPOC y una elevada tasa de infradiagnóstico, encontrando que el 73\% de los pacientes estaba sin diagnosticar y la mayoría de los que se diagnosticaban ya se encontraban en estadios III y IV (2). Este retraso en el diagnóstico, debido al carácter progresivo e irreversible de la enfermedad provoca una elevada morbilidad, así como un elevado coste asistencial. Por todo esto es necesario un diagnóstico precoz (2-4).

Las actuales guías de abordaje de la EPOC como GOLD 2011 (3) o GesEPOC (4) recomiendan mecanismos para detectar a los pacientes en las primeras fases de la enfermedad, que es cuando se puede sacar más partido a determinados tratamientos, y que estos sean más costo-efectivos. También queda patente esta preocupación en el Plan Estratégico Nacional (5) donde se recoge que, ante la inviabilidad de cribados masivos, la alternativa más eficiente (coste-efectiva) pasa por seleccionar poblaciones de alto riesgo y emplear en ellas pruebas diagnósticas que cumplan con requisitos mínimos de sensibilidad, reproductibilidad y validación. Aconsejando que se debe sospechar EPOC en todo individuo mayor de 40 años, que tenga factores de riesgo, como exposición al humo de tabaco $(>10$ paquetes/año), y que presente sintomas compatibles con la enfermedad (tos, expectoración, disnea de esfuerzo). Este diagnóstico deberá confirmarse mediante la práctica de una espirometría que demuestre la existencia de limitación al flujo aéreo (5).

Para realizar técnicas de cribado contamos con cuestionarios de síntomas y medidores de función pulmonar más sencillos, dejando la espirometría como prueba de confirmación diagnóstica (6). En España sólo tenemos validado al castellano el COPD-PS (7) que incluye 5 preguntas sobre síntomas, hábito tabáquico y edad del paciente. Existen también dispositivos portátiles de medición de parámetros respiratorios validados que se pueden utilizar solos o junto a los cuestionarios, como el Vitalograph COPD-6 $(8,9)$ (figura 1).

Siguiendo esta línea se están realizando experiencias de cribado en diferentes ámbitos, y por diferentes profesionales sanitarios (10), por supuesto también en farmacias (11-13). El proyecto más importante de este tipo es el FARMAEPOC II (14), que al cierre de este estudio sólo había publicado los resultados preliminares, organizado por la SEPAR y el Colegio Oficial de Farmacéuticos de Barcelona, y donde participan 100 farmacias comunitarias. Se ha de resaltar que este servicio de detección precoz de EPOC en farmacia comunitaria se viene realizando a nivel internacional desde hace más de diez años, estando ya plenamente implantado en otros países (15).

\section{Hipótesis de estudio}

¿Podría disminuir el infradiagnóstico de la EPOC en nuestra comunidad implantando en las farmacias comunitarias un servicio de detección precoz? La farmacia comunitaria, por razones de accesibilidad, de preparación del farmacéutico y confianza del paciente, podría jugar un importante papel en el diagnostico precoz de esta enfermedad realizando funciones de cribado, y derivación al personal médico, con la realización de una sencilla prueba de función pulmonar acompañada de la realización de un test rápido $(5,11-14)$.

\section{Objetivos \\ Principal}

Identificar pacientes de EPOC no diagnosticados, mediante un servicio de cribado de EPOC ofrecido por

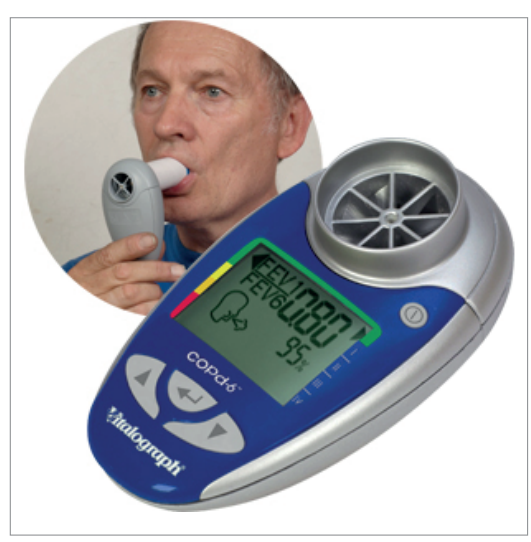

Figura 1. Dispositivo electrónico Vitalograph COPD-6

farmacias comunitarias de Baleares a través de un circuito de diagnóstico precoz, y un protocolo de derivación a atención primaria.

\section{Secundarios}

1. Evaluar el servicio de cribado de EPOC ofrecido por las farmacias comunitarias de Baleares, su viabilidad y eficiencia, mediante la correlación entre el resultado del diagnóstico precoz en la farmacia y el resultado confirmatorio del diagnóstico de EPOC.

2. Caracterizar la población no diagnosticada susceptible de padecer EPOC en Baleares.

\section{Material y métodos \\ Diseño y emplazamiento}

Se trata de un estudio descriptivo, transversal y multicéntrico, dirigido por la delegación balear de la Sociedad Española de Farmacia Familiar y Comunitaria (SEFAC IB), con la colaboración de la "Associació Balear de Medicina Familiar i Comunitaria” (AMFIC), realizado en 30 farmacias comunitarias que disponían de una zona de atención personalizada, y en el que participaron 42 farmacéuticos que previamente habían recibido formación en métodos de cribado de EPOC. Previamente al inicio del estudio se realizó la correspondiente promoción y divulgación del mismo entre los centros de salud cercanos a las farmacias participantes, médicos de AP, y Gerencia de Atención Primaria de Mallorca.

\section{Material}

Cuestionario COPD-PS en español validado por Miravitlles et al. en 
2011 (7). Se establece como punto de corte para este estudio de cribado la puntuación de 4, ya que ofrece una mayor sensibilidad que el 5 (93,59 vs $65,38 \%$ ) aún a costa de una menor especificidad (64,84 vs 87,91\%) (7). Dispositivo portátil electrónico Vitalograph COPD-6 validado en cribado de EPOC en atención no especializada en diferentes estudios $(6,8,9)$. El punto de corte utilizado en el estudio para el cociente FEV1/FEV6 es de 0,75 , siguiendo las recomendaciones de estudios de referencia para cribado de EPOC (7-9)

\section{Población de estudio}

Criterios de inclusión: Mayores de 40 años de ambos sexos, fumadores (de $\geq 10$ paquetes/año, que son los que presentan el riesgo), exfumadores (considerando como tales a los que llevan más de un año sin fumar) y fumadores pasivos (trabajan o conviven con fumadores), que en el cuestionario COPD-PS obtuvieron una puntuación $\geq 4$ y que voluntariamente aceptaron participar en el estudio y firmar el consentimiento informado. Los pacientes que cumplían todos los criterios de inclusión se incluyeron en el estudio y pasaron a realizar la prueba de expiración forzada con el COPD-6.

Criterios de exclusión: pacientes diagnosticados de EPOC, asma $u$ otras enfermedades respiratorias, en tratamiento crónico con fármacos de uso en enfermedad respiratoria $u$ otras enfermedades que puedieran enmascarar el resultado de la prueba. Personas que por sus condiciones psíquicas o físicas no pudieran realizar correctamente la prueba respiratoria o responder al cuestionario y podrían ser potenciales pérdidas del estudio. Pacientes incapaces de leer, entender, o firmar correctamente la hoja de información al paciente y el consentimiento informado. Embarazadas.

Circuito y protocolo de derivación (figura 2)

El ofrecimiento del servicio se reforzó con la edición de carteles que se distribuyeron entre las farmacias participantes. Se planteó la posibilidad de participar en el estudio a los pacientes que acudian a la farmacia para retirar su medicación, y/o demandar el servicio de indicación

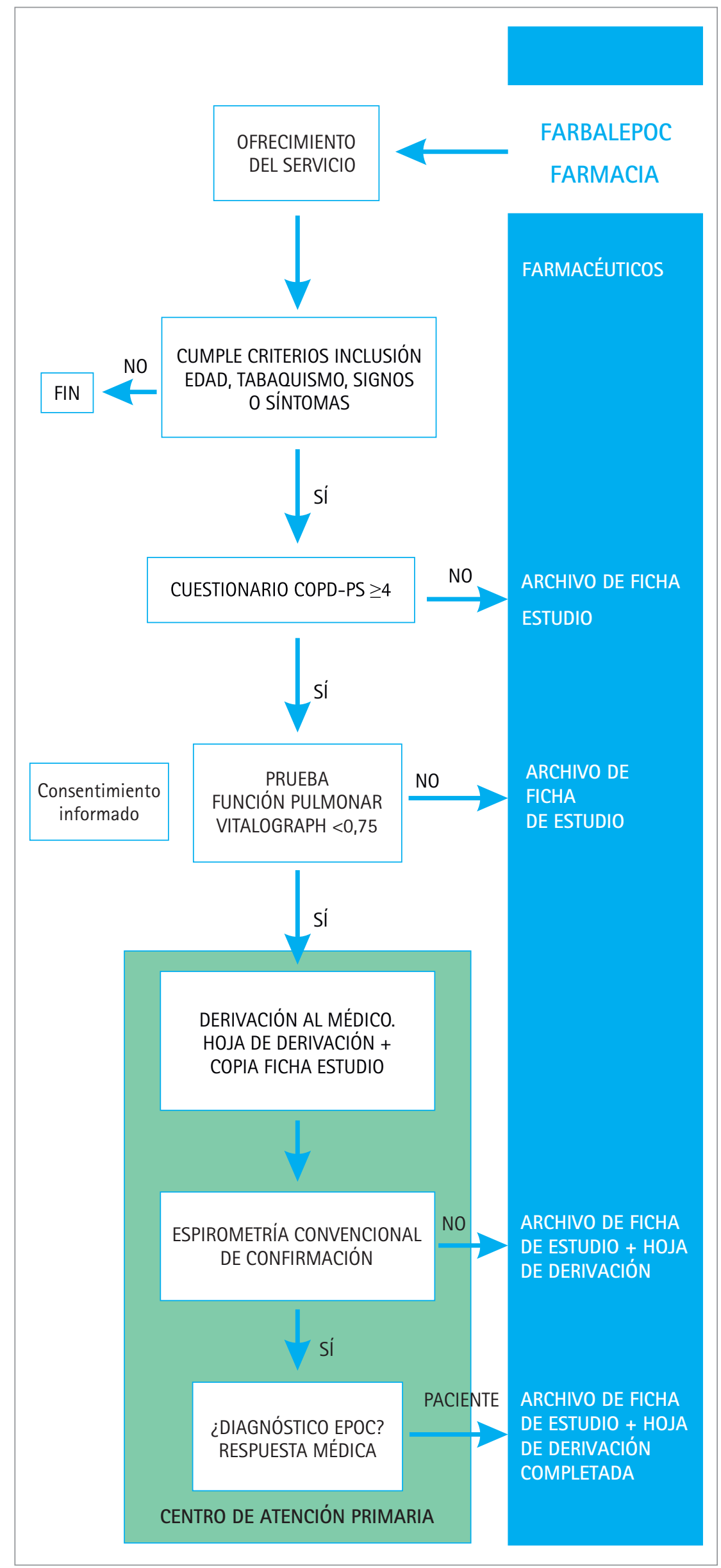

Figura 2. Algoritmo para el cribado de EPOC utilizado en el estudio 
farmacéutica, y que cumplían los criterios de inclusión firmando el documento de consentimiento informado. Si la puntuación obtenida en el cuestionario era $\geq 4$ puntos, se les realizaba la prueba de función pulmonar mediante la expiración forzada, anotando en el documento a tal efecto el mejor resultado del cociente FEV1/ FEV6, de las tres pruebas realizadas consideradas válidas. Si el valor obtenido del cociente FEV1/FEV6 era $<0,75$ se derivaba el paciente a su médico de familia con una copia de la ficha de estudio y la hoja de derivación. La otra copia de cada documento quedaba en poder del farmacéutico participante para minimizar posibles pérdidas y para su posterior estudio $\mathrm{y}$ análisis.

La fase de estudio inicial fue de 4 meses, prolongándose 3 más para la recopilación de datos de las derivaciones a AP, del 29 de noviembre al 30 de junio.

Variables principales del estudio

- Cociente FEV1/FEV6 <0,75 medido con el Vitalograph COPD-6.

- Cociente FEV1/FEV6 medido con espirometría forzada con prueba broncodilatadora realizada en AP.

- Confirmación diagnóstico de EPOC en AP.

Variables secundarias

- Datos demográficos, edad y sexo.

- Resultados del test COPD-PS.

- Hábito tabáquico: fumador (persona que fuma como mínimo 10 paquetes al año), exfumador (persona que haya sido fumadora pero que lleve como mínimo un año sin fumar ningún cigarrillo), fumador pasivo (persona que no ha fumado de forma regular, pero que convive o trabaja junto a fumadores regularmente).

- Historia tabáquica: número de cigarrillos/día, años de fumador, años de abstinencia, años de exposición.

- Signos y sintomas crónicos: presencia de sintomas respiratorios más habituales de EPOC (tos, expectoración y disnea) de forma habitual.

- Edad pulmonar o capacidad pulmonar aproximada, expresada en años.

\section{Tamaño muestral}

La muestra teórica se estableció en 191 individuos con un nivel de confianza del 95\%, una precisión del 5\% y una tasa de reposición por posibles pérdidas de participación o no finalización del estudio, estimada del 15\% (con una tasa de reposición nula la muestra teórica sería de 166 individuos). Los cálculos se basan en la población balear de entre 40 a 80 años (484.285 individuos), y con base en una prevalencia de EPOC del 10,2\% (2).

\section{Análisis estadístico}

Las variables continuas fueron descritas mediante la mediana y el rango intercuartílico. Se comprobó la asunción de normalidad para todas las variables continuas mediante prueba de Shapiro-Wilk y gráfico Q-Q. Para describir las variables categóricas se utilizaron frecuencias relativas.

Se utilizaron pruebas no paramétricas y la prueba chi-cuadrado para comparar variables continuas y categóricas, respectivamente. Todas las pruebas estadísticas fueron de 2 colas y con el nivel de significación estadística fijado en el 0.05 y ajustado por la corrección de Bonferroni por comparaciones múltiples.

Se usaron correlaciones no paramétricas para relacionar variables continuas.

Se utilizó el análisis de regresión logística para identificar variables asociadas con el riesgo de ser susceptible de padecer EPOC, controlando por sexo y edad.

Todos los análisis fueron realizados con SPSS ${ }^{\circledast}$ 19.0.

\section{Resultados}

Participaron 24 farmacias, registrándose 198 casos, la media de casos enviados por farmacia fue de 8,3 , realizando la prueba de expiración forzada a 154 pacientes $(6,4)$ farmacia) que son los que obtuvieron valores iguales o mayores a 4 en el cuestionario COPD-PS. Por islas, 127 casos (un 82,5\% del total) son de Mallorca, 11 (un 7,7\%) de Menorca y 16 (un 10,3\%) de Formentera. La mediana de edad fue de 56 años (IQR=49,0; 64,0), 76 hombres $(49,4 \%)$ y $78 \mathrm{mu}-$ jeres $(51,6 \%)$.

La mayoría de los pacientes $(69,5 \%)$ eran fumadores $(n=107)$ con mayor proporción de mujeres fumadoras $(56,1 \%)$, seguidos de exfumadores $(n=38)(24,7 \%)$ con mayor proporción de hombres $(68,4 \%)$, fumadores pasivos $(n=4)(2,6 \%)$ y exfumadores/fumadores pasivos $(n=5)$ $(3,2 \%)$. Los pacientes considerados como fumadores $(n=107)$ presentaron una mediana de 33 años fumando (IQR=27,0; 40,0) y una mediana de 20 cigarrillos/día (IQR=16,0; 30,0). Los pacientes considerados exfumadores $(n=43)$ fumaron una mediana de 25 años (IQR=15,0; 40,0), una mediana de 20 cigarrillos/día (IQR=15,0; 30,0) y hace una mediana de 13 años que no fuman (IQR=4,5; 26,0).

En cuanto a los signos o síntomas que presentaban los pacientes: 91 $(59,1 \%)$ decían presentar expectoración, $87(56,5 \%)$ manifestaban padecer tos y 85 (55,2\%) disnea como síntomas más destacados. En 93 (60,3\%) casos, los pacientes presentaban más de un sintoma o signo simultáneamente.

Los resultados del cuestionario COPD-PS tuvieron una mediana de 5 (IQR=4,0; 5,0) con un mínimo de 4 puntos y un máximo de 9 .

Los resultados de la prueba de expiración forzada FEV1/FEV6 obtuvieron un cociente de mediana de 0,79 (IQR=0,74; 0,87), un mínimo de 0,24 y un máximo de 1.

La mediana de la edad pulmonar calculada por el COPD-6 fue de 72 (IQR=58,5; 83,0).

$38(24,7 \%)$ de los 154 pacientes presentaron resultados del cociente FEV1/FEV6 inferiores a 0,75, y se les derivó a su médico de atención primaria para que realizase la espirometría de referencia y confirmar el posible diagnóstico de EPOC (tabla 1). En ese momento $8(21,1 \%)$ de ellos manifiestan al farmacéutico participante su intención de no acudir a su médico en el plazo de duración del estudio por diferentes motivos. Por lo tanto fueron finalmente 30 los pacientes realmente derivados.

La prevalencia de posible EPOC $(n=38)$ en este estudio se ha estimado en un 19,2\% de los pacientes encuestados $(n=198)$. Por islas, se detectaron 29 (76,3\%) en Mallorca, 5 (13,1\%) en Menorca y $4(10,5 \%)$ en Formentera. La mediana de edad fue de 60,5 años (IQR=54,0; 66,5). Por sexos, 18 $(47,4 \%)$ fueron hombres y $20(52,6 \%)$ mujeres. La mediana de años fumando de los pacientes fumadores $(n=28$, 
un $73,7 \%$ del total) fue de 38 años $(\mathrm{IQR}=30,0 ; 41,5)$, la mediana de cigarrillos/día fue de 20 (IQR=15,0; 30,0). La mediana de años fumando de los exfumadores ( $n=6$, un $15,8 \%$ del total) fue de 30 años (IQR=10,5; 42,5), la media de cigarrillos/día que fumaba fue también de 20 (IQR=7,5; 25,0). La mediana de años que ya no fumaban fue de 10 (IQR=2,0; 19,0) años. Además se consideraron también 4 $(10,6 \%)$ pacientes como fumadores pasivos habiendo sido la mitad de ellos también exfumadores. De todos ellos $(\mathrm{n}=38), 27(71,1 \%)$ presentaban tos, $19(50,0 \%)$ expectoración y 26 $(68,4 \%)$ disnea. En 25 (65,7\%) casos los pacientes presentaban más de un signo o síntoma. La totalidad de los signos y sintomas se presenta en la figura 3.

En cuanto al resultado del test COPD-PS de los casos positivos, la puntuación mediana fue de 5 (IQR $=4,0 ; 6,0)$, la puntuación mediana del cociente FEV1/FEV6 fue de 0,69 (IQR=0,62;0,73) y la mediana de edad pulmonar fue de 88 años (IQR=78,0; 102,0).

De los pacientes susceptibles de padecer EPOC $(n=30)$ y correctamente derivados a Atención Primaria según el circuito creado, se recopila en la farmacia el posible diagnóstico de EPOC en 17 (56,6\%) de ellos. En 16 $(94,1 \%)$ de los cuales se confirma el diagnostico de EPOC, siendo únicamente un caso no considerado por el médico como paciente de EPOC. En los $13(43,4 \%)$ casos restantes al cierre del estudio se seguía a la espera de conocer la resolución de sus casos.

De los pacientes susceptibles de ser diagnosticados de EPOC $(n=38)$ únicamente se ha cerrado todo el circuito con los resultados de la espirometría de confirmación en manos del farmacéutico participante, en 5 $(13,2 \%)$ casos (en el $80 \%$ de los casos se confirmaron los resultados de la prueba realizada en la farmacia y se diagnosticó EPOC).

En cuanto a las diferentes asociaciones y relaciones entre las variables estudiadas:

Se ha podido constatar que los pacientes susceptibles de presentar EPOC $(n=38)$ presentan una mediana de edad (60,5 años) superior a la que presentan el total de los pacientes incluidos en el estudio ( $n=154)$ (56 años). A partir de una regresión logística se puede estimar como

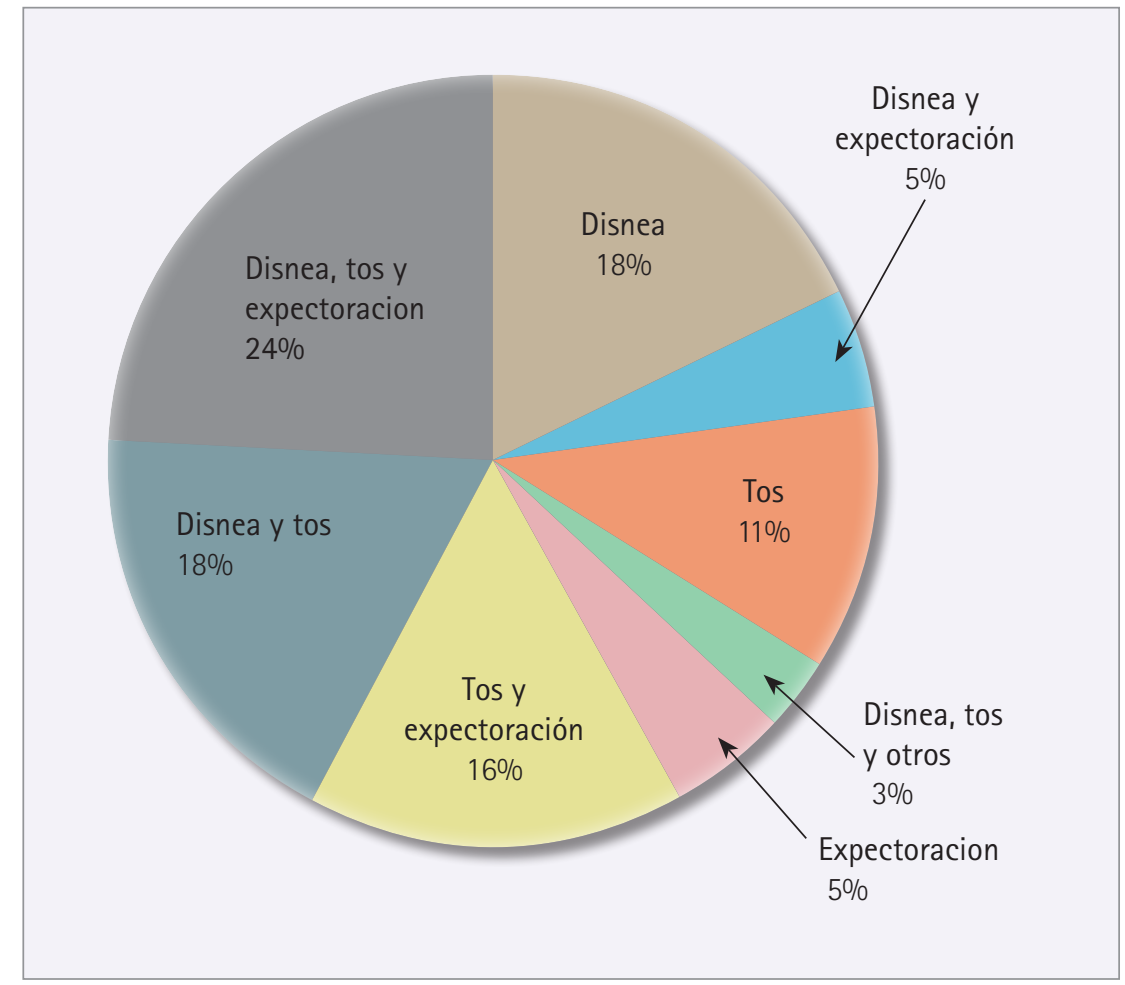

Figura 3. Signos y síntomas crónicos de pacientes con valores de FEV1/FEV6 <0,75

aumenta el riesgo de ser susceptible de padecer EPOC con la edad, un $8,6 \%$ por cada año adicional (IC95\% odds-ratio $=0,5 \% ; 17,5 \%)$. También se ha podido constatar que los pacientes fumadores del grupo susceptible de presentar EPOC presentan una mediana de años fumando (38 años) superior a la del total de pacientes del estudio (33 años). Aunque esta asociación no ha sido estadísticamente significativa $(p=0,87)$. Sí que se ha podido encontrar una asociación estadísticamente significativa entre presentar tos o disnea crónica y el riesgo de presentar un valor del cociente FEV1/FEV6 $<0,75$ en la farmacia ( $p=0,00$ en ambos casos). En concreto, los pacientes con tos crónica presentan un riesgo 10,4 veces mayor de presentar valores $<0,75$ en la prueba de expiración forzada en la farmacia (IC95\% odds-ratio $=2,4$; $45,5)$, mientras que los pacientes con disnea presentan un riesgo 5,9 veces mayor de presentar valores $<0,75$ en la misma prueba (IC95\% odds-ratio $=1,7 ; 20,3$ ).

En cuanto a la puntuación del cuestionario COPD-PS, por cada punto adicional obtenido, el riesgo a ser diagnosticado de EPOC aumenta de media un 63\% (IC95\% odds-ratio $=9,3 \% ; 143,0 \%)$. Va en la misma línea la relación entre puntuación del COPD-PS y resultado del cociente
FEV1/FEV6 de la prueba de expiración forzada realizada en la farmacia, presenta una correlación negativa $(\mathrm{r}=-0,2 ; \mathrm{p}=0,016)$, a mayor puntuación del cuestionario, menor es el resultado obtenido de la prueba de expiración forzada.

\section{Discusión}

Este estudio ha presentado algunas limitaciones coincidentes con las de otros estudios multicéntricos. Si bien se han registrado 198 casos, solamente 154 registraron un resultado del test COPDPS $\geq 4$, y pasaron a realizar la prueba de expiración forzada en la farmacia. En consecuencia no se ha conseguido alcanzar el tamaño muestral inicialmente previsto (154 vs 166), debido a que en lugar de 30 farmacias, tan solo participaron realmente en el estudio 24 , siendo también la media de casos válidos por farmacia más baja de lo esperado $(6,4$ vs 10).

En cuanto al objetivo principal del estudio que era identificar pacientes de EPOC no diagnosticados, se ha cumplido ya que como mínimo se ha registrado que 16 pacientes que desconocían padecer la enfermedad se han diagnosticado y tratado correctamente gracias a su participación en él.

En cuanto a evaluar la viabilidad y eficiencia del servicio mediante el 
circuito, y protocolo de derivación diseñado entre las farmacias comunitarias y los centros de atención primaria, de los $38(24,7 \%)$ pacientes susceptibles de padecer EPOC, que sepamos, solamente 16 se han podido diagnosticar y tratar adecuadamente al cierre del estudio, y tan solo en 5 casos se ha realizado correctamente todo el circuito con la devolución al farmacéutico de la hoja de derivación correctamente cumplimentada por el médico de atención primaria. En los otros casos positivos, se han confirmado indirectamente, a través de los mismos pacientes, mediante el uso del aplicativo de receta electrónica del "Servei Balear de Salut" o por comunicación directa con el médico. Por la tanto no se ha podido correlacionar el resultado del diagnóstico precoz en la farmacia y el resultado confirmatorio del diagnóstico de EPOC por espirometría por falta de muestra. Por otro lado son cifras semejantes, e incluso algo mejores que otros estudios similares publicados, pudiéndose concluir que los circuitos y protocolos de derivación son las grandes limitaciones de estos proyectos (11-14). Las debilidades del circuito diseñado han sido de diferentes tipos, en general han afectado a pacientes, farmacéuticos, médicos y a la propia estructura de funcionamiento del "Servei Balear de Salut". En primer lugar, como se ha comentado, la implicación de los farmacéuticos participantes ha estado por debajo de lo esperado en la mayoría de los casos. En segundo lugar, si nos referimos a los pacientes, $8 \mathrm{de}$ ellos no han querido derivar su caso al médico de atención primaria, ha sido una tasa de pérdida superior a la esperada (21\% vs 15\%). En la participación de los centros de atención primaria se ha evidenciado en general la falta de cooperación de la mayoría de médicos implicados, a pesar de la promoción realizada del estudio entre los médicos y coordinadores de los centros y de la hoja de derivación que les entregaban los pacientes con la información específica e instrucciones a seguir. Esta situación podría haberse resuelto si los médicos y centros de salud participantes no hubiesen sido elegidos de forma aleatoria sino que hubiesen participado médicos voluntarios, motivados y formados en el proyecto.

En cuanto a la caracterización de los pacientes susceptibles de padecer
EPOC, la prevalencia se ha estimado según este estudio en un $19,2 \%$ de la población de riesgo (fumadores, exfumadores y/o fumadores pasivos) en concordancia con diferentes estudios publicados $(1,2)$ (20-25\%), sin diferencias significativas en cuanto al sexo. También se ha podido comprobar en coincidencia con los mismos estudios $(1,2)$ que el riesgo de presentar EPOC aumenta con la edad, con el número de años que se haya fumado, en pacientes que presentan signos o síntomas crónicos como tos y disnea, y cuanto más elevada es la puntuación obtenida en el cuestionario COPD-PS.

Todos estos datos y relaciones entre variables refuerzan el correcto diseño del estudio en cuanto a los criterios de inclusión, y a los puntos de corte usados para derivar a los pacientes a la realización de la espirometría de confirmación, con el objetivo de lograr un servicio de cribado de EPOC con la mejor relación entre sensibilidad/especificidad y coste/ efectividad.

El significativo porcentaje de pacientes a los se les ha detectado una posible EPOC (24,7\%), en línea con el elevado grado de infradiagnóstico detectado en el estudio EPISCAN indican que sería muy importante y hasta cierto punto necesario la implantación de servicios de cribado y detección precoz de EPOC en las farmacias comunitarias. Todo ello centrado en los pacientes fumadores o exfumadores, contribuyendo con este servicio a la concienciación sobre los riesgos del tabaco y al aumento en el porcentaje de cesación tabáquica.

Por otro lado es obvio que aunque el método y materiales usados en el estudio cumplen los criterios de viabilidad, el circuito creado no es eficiente y es muy mejorable en la actuación de todos sus actores, siendo este el verdadero talón de Aquiles de este tipo de estudios.

\section{Agradecimientos}

A Javier Plaza Zamora, farmacéutico comunitario de Murcia, excoordinador del Grupo de Respiratorio de SEFAC. Asesor del estudio.

Xavier Grimal Agell. Economista. Responsable del tratamiento estadístico y analítico de los resultados.

Natalia Rapado Peña. Farmacéutica. Regional Market Access. Departa- mento de Relaciones Institucionales. Laboratorios Almirall. Impulsora del estudio.

Laboratorios Almirall, por su apoyo económico en la compra de materiales y fungibles.

A las 24 farmacias participantes por orden de participación: Sanz Guillén, Bennasar Bibiloni, Noguera Pascual, García de Vena, Hernández-Hernández, De Eguileor Lasquibar, M. Pons-B. Pons, J. Frau-C. Frau, Sitjar Garí, Guitart Lorente de No, Oliver Oliver, Palmer Llaneras, Pons Sanz, Alemany Reus, Aguiló Juanola, Serra Farell, Fuster Arnau, Ubach Turull, Llull Cabrer, Costa Amengual, Bonnin Sánchez, March Balle, Seguí Puntas, Martí Sureda.

\section{Referencias bibliográficas}

1. Sobradillo V, Miravitlles M, Jimenez CA, Gabriel R, Viejo JL, Masa JF, et al. Estudio IBERCOP en España: prevalencia de síntomas respiratorios habituales y de limitación crónica al flujo aéreo. Arch Bronconeumol 1999;35:159-66. doi:0.1016/S03002896(15)30272-6

2. Miravitlles M, Soriano JB, García-Río F, Muñoz L, Duran-Taulería E, Sánchez G, et al. Prevalence of COPD in Spain: Impact of undiagnosed COPD on quality of life and daily life activities. Thorax 2009;64:863-868. doi:10.1136/thx.2009.115725

3. Global Initiative for Chronic Obstructive Lung Disease (GOLD). Global Strategy for the Diagnosis, Management, and Prevention of Chronic Obstructive Pulmonary Disease (Revised 2013). [sede Web]. GOLD 2011 [acceso 20-9-2013]. Disponible en: http:// www.goldcopd.org.

4. Guía de Práctica Clínica para el Diagnóstico y Tratamiento de de Pacientes con Enfermedad Pulmonar Obstructiva Crónica (EPOC) - Guía Española de la EPOC (GesEPOC). Arch Bronconeumol 2012;48(Supl 1):2-58. doi:10.1016/S0300-2896(12) 70035-2

5. Estrategia en EPOC del Sistema Nacional de Salud. Plan de Calidad para el Sistema Nacional de Salud. Ministerio de Sanidad y Política Social; 2009. [sede Web]. [acceso 21-9-2013]. Disponible en: http://www.msssi.gob. es/organizacion/sns/planCalidadSNS/ docs/EstrategiaEPOCSNS.pdf

6. Calvo Corbella E. Métodos de detección de casos de enfermedad pulmonar obstructiva crónica en atención primaria. Pubepoc 2011;1:5-14.

7. Miravitlles M, Llor C, Calvo E, Díaz S, Díaz-Cuervo H, González-Rojas N. 
Validación de la versión traducida del chronic obstructive pulmonary disease-population screener (COPD-PS). Su utilidad y la del FEV1/FEV6 para el diagnóstico de EPOC. Medicina clínica 2012; 139(12):522-530. doi:10.1016/j. medcli.2011.06.022

8. Del Olmo Zamora R, Martín Herruzo AI, Gutierrez Cabanas M, ¿Podemos utilizar el dispositivo COPD 6 como screening de la EPOC en atención primaria? Revista Andaluza de Atención Primaria 2013;2(Supl 2):34.

9. Represas Represas C, Botana Rial M, Leiro Fernández V, González Silva AI, Del Campo Pérez V, Fernandez-Villar A. Validación del dispositivo portátil COPD-6 para la detección de patologías obstructivas de la vía aérea. Arch Bronconeumol 2010;46:426-32. doi:10.1016/j.arbres.2010.04.008
10. Clotet J, Real J, Lorente I, Fuentes A, Paredes E, Ciria C. Espirometria como método de cribado y de intervención antitabaco en fumadores de alto riesgo en atención primaria. Aten. Prim. Jun 2012;44(6):328-334. doi:10.1016/j.aprim.2011.05.012

11. Castillo D, Guayta R, Giner J, Burgos F, Capdevila C, Soriano J.B, et al. COPD case finding by spirometry in high-risk customers of urban community pharmacies: A pilot study FARMAEPOC group. Respir Med 2009;103:839-845. doi:10.1016/j. rmed.2008.12.022

12. Segovia Sánchez M, Vila Sosa MA, Trave Mercade P, March Pujol M. Cribaje de la EPOC desde la farmacia comunitaria: protocolo y resultados del estudio. Farmacéuticos Comunitarios 2012;4(1):15-21.
13. Plaza FJ, Sabater M, Garrido $M$, Peña JE, Sánchez D, Clavel A. Detección de pacientes susceptibles de padecer EPOC en farmacias comunitarias de la Región de Murcia. Farmacéuticos Comunitarios 2014;6(Supl 1):43-44.

14. Castillo Villegas D, Burgos F, Giner J, Estrada M, Soriano JB, Flor X, et al. Cribado de EPOC: Nuevas herramientas y circuitos sanitarios. Resultados preliminares del FARMAEPOC 2. Arch Bronconeumol 2011;(4):9192.

15. Fuller L, Conrad WF, Heaton PC, Panos R, Eschenbacher W, Frede SM. Pharmacist-managed chronic obstructive pulmonary disease screening in a community setting. J Am Pharm Assoc 2003;52(5):5966. 